ISSN 0103-8478

\title{
Turboatomizador e repasse com pistola manual na cobertura de pulverização de agrotóxicos em caquizeiro (Diospyros kaki L.f.)
}

\author{
Air-jet sprayer and spray gun in the pesticide spray coverage of persimmon \\ (Diospyros kaki L.f.) trees \\ Leandro Riyuiti Higashibara $^{{ }^{*}}$ Viviane Dutra $^{\mathrm{I}}$ Otavio Jorge Grigoli Abi Saab $^{\mathrm{II}}$ \\ Hideaki Wilson Takahashi" ${ }^{\text {II }}$ Carmen Sílvia Vieira Janeiro Neves ${ }^{\text {II }}$
}

RESUMO

A cultura do caquizeiro vem crescendo em importância no Brasil e, com o aumento do cultivo, tem havido também incremento dos problemas relacionados a doenças $e$ pragas. A pulverização de agrotóxicos é o método mais utilizado na prevenção e no controle desses problemas e a principal forma de aplicação é a pulverização hidráulica com auxilio de fluxo de ar. $O$ objetivo deste trabalho foi avaliar o efeito da variação da taxa de aplicação e do repasse na parte interna das plantas em pulverização com pistola manual, na cobertura de calda das folhas. A pulverização foi realizada em plantas de caquizeiro com 17 anos, utilizando-se equipamento composto de trator $e$ pulverizador turboatomizador. A aplicação foi realizada com duas velocidades de avanço do equipamento, o que promoveu diferentes taxas de aplicação. Para a realização do repasse, utilizou-se pistola manual acoplada ao mesmo equipamento. Para a avaliação da cobertura, utilizou-se calda preparada com corante fluorescente dissolvido em água. Foram coletadas folhas nas posições externa e interna das plantas. Posteriormente, em sala escura e com iluminação ultravioleta para destacar a área atingida pelo corante fluorescente, as superfícies abaxial e adaxial de cada folha foram fotografadas com câmera digital. Cada imagem gerada foi submetida à análise pelo software SIARCS 3.0, resultando em porcentagem de cobertura. $O$ delineamento experimental utilizado foi de blocos ao acaso em parcelas subsubdivididas com quatro repetições, sendo os tratamentos arranjados em esquema fatorial (taxas alta e baixa de aplicação, com e sem repasse manual, localizações interna e externa da folha na planta). Foi avaliada a cobertura das superfícies abaxial e adaxial das folhas. Houve interação significativa entre tratamentos (taxa de aplicação e repasse manual), posição e superfície das folhas. A maior taxa de aplicação promoveu aumento significativo da cobertura na superficie abaxial das folhas, independentemente da posição destas na planta (interna ou externa). Houve efeito do repasse com pistola na cobertura da superfície abaxial das folhas situadas na posição interna, mas não na externa à planta. Considerando que a maioria dos agentes causadores de danos fitossanitários inicia a ocorrência na superficie abaxial das folhas, pode-se concluir que o repasse manual foi mais eficiente do que o aumento da taxa de aplicação, visando à cobertura nas folhas posicionadas no interior da planta.

Palavras-chave: Taxa de aplicação, tecnologia de aplicação, produtos fitossanitários.

\begin{abstract}
Persimmon crop is growing in importance in Brazil, and with the increase of the production area there also has been an increase in the problems related to diseases and pests. For the control and prevention of these problems, most often it is used pesticides spray, and one of the main techniques is the spray with the aid of air flow. The objective of this study was to evaluate the effects of changes in the application rate and transfer with a spray gun on the leaves mixture coverage. The spraying was performed using tractor and air flow sprayer. The application was performed with two forward speeds of the equipment, leading to different application rates. The transfer was made with a spray gun attached to the same equipment. For the assessment of the coverage it was used a mixture prepared with fluorescent tracer dissolved in water. Leaves were collected in the inside and outside of the tree. Later, in dark room with ultraviolet lighting to highlight the area affected by the fluorescent tracer, the upper and lower surfaces of each leaf were photographed with a digital camera. Each image was subjected to analysis generated by software SIARCS 3.0, resulting in percentage of coverage. It was used a randomized block design with subdivided parcels with four replications, being the treatments in a $2 \times 2 \times 2$ factorial scheme (high or low application rate, with or without transfer, inside or outside the tree). The evaluations were made in the upper and lower leaf surfaces. There was significant interaction between treatments (application rate and spray gun transfer), leaf position
\end{abstract}

'Programa de Pós-graduação em Agronomia, Centro de Ciências Agrárias (CCA), Universidade Estadual de Londrina (UEL), CP 6001, 86051-970, Londrina, PR, Brasil. E-mail: riyuitih@ hotmail.com. *Autor para correspondência.

"Departamento de Agronomia, CCA, UEL, Londrina, PR, Brasil. 
and surface. High application rate promoted a significant increase in the coverage of the lower leaves, regardless the site in the plant (inside or outside). The inside leaves had a better coverage with the spray gun transfer. As most of the agents of plant damage begin to occur in the lower surface of leaves, it can conclude that the spray gun transfer was more effective to cover the leaves inside the plant than increasing the application rate.

Key words: Application rate, spray technology, fungicide.

\section{INTRODUÇÃO}

A cultura do caquizeiro vem crescendo em importância no Brasil. Segundo o IBGE (2009a), a produção brasileira dessa fruta correspondente a 171 mil toneladas/ano, sendo destaque o Estado de São Paulo, com uma produção aproximada de 85 mil toneladas (IBGE, 2009b). No Paraná, a produção em 2009 foi de 23 mil toneladas (IBGE, 2009c).

A ocorrência de problemas fitossanitários é um dos entraves na produção de caqui em muitos países (WILLIAMSON \& SUTTON, 2010). No Brasil, com o aumento do cultivo, houve também incremento da incidência de doenças e pragas que podem diminuir a produtividade e a longevidade dos pomares, sendo a aplicação de agrotóxicos a medida mais utilizada para controle e prevenção desses problemas. O uso de fungicidas, aplicados de forma preventiva, é útil para evitar a propagação de inóculos viáveis nas plantas sadias (BUCK et al., 2011). A aplicação em pulverização pode ser feita manualmente, por meio de pistolas, ou com equipamento tracionado e acionado por trator. Uma das técnicas bastante utilizada para a aplicação é a pulverização hidráulica com auxílio de fluxo de ar. O uso apropriado de assistência de ar é exigido para melhorar a distribuição de depósitos e a eficiência da coleta da calda aplicada pelas diversas partes da planta (MATTHEWS, 2000).

Para a cultura do caquizeiro, não há registro de dados que auxiliem os produtores a decidir sobre a melhor forma de proteger a cultura, permanecendo a dúvida sobre a eficácia dos equipamentos com auxílio de fluxo de ar, conhecidos comercialmente como turboatomizadores, em relação à aplicação com pistolas manuais. Outra questão é quanto à eficiência do repasse, que é usado por muitos produtores, e que consiste na aplicação com pistola manual direcionada ao interior da planta, de baixo para cima, como técnica auxiliar na proteção das partes internas da planta, mais suscetíveis ao ataque de pragas e doenças.

É indispensável que a assistência de ar vença a distância entre o pulverizador e o alvo pretendido, sendo que o volume de ar deve ser ajustado às circunstâncias específicas da aplicação, visando a reduzir as perdas ocorridas quando o produto não alcança o alvo, ou o ultrapassa (KAUL et al., 2002). Os turboatomizadores são os pulverizadores mais utilizados em frutíferas, entretanto, uma parte significativa das aplicações em pomares não é depositada no alvo, mas perdida no ar e no solo, implicando danos econômicos e poluição ambiental (HOLOWNICKI et al., 2000).

O fluxo do ar nos turboatomizadores, volume de ar gerado na turbina por tempo, influencia diretamente na distribuição vertical do líquido aplicado. O fluxo de ar tem como objetivo favorecer a trajetória das gotas pulverizadas em direção ao alvo, interferindo em sua penetração e consequente deposição (GIL et al., 1996).

Em experimento realizado em árvores frutíferas, para uma mesma potência despendida, o maior volume de ar, a uma baixa velocidade aerodinâmica, propiciou melhor penetração da calda e produziu melhor cobertura de folha do que baixos volumes de ar, com velocidade mais alta (RANDALL, 1971). Essa constatação é verdadeira, contanto que a velocidade aerodinâmica do ar seja alta o suficiente para formar aberturas para a corrente de ar penetrar. A presença de mais ar não implica necessariamente uma cobertura melhor.

Em outro trabalho realizado em pomares, o depósito de calda total foi melhor quando a taxa de fluxo de ar foi baixa, enquanto que, para uma taxa maior, foi aumentada a quantia de gotas atomizadas sopradas através da cultura (HOLOWNICKI et al., 2000). Esses resultados foram reproduzidos quando a velocidade do vento era baixa. Mas, em velocidades mais altas, os depósitos eram mais baixos nas taxas menores (CROSS et al., 2001).

O objetivo deste trabalho foi avaliar os efeitos da variação da taxa de aplicação e do repasse em pulverização com pistola manual na parte interna das plantas, na cobertura das folhas de caquizeiro.

\section{MATERIAL E MÉTODOS}

O trabalho foi realizado numa área de produção comercial de caqui, com total aproximado de 3,6ha, localizada no município de Faxinal/PR (235ㄷ,553'S, 51 $\left.{ }^{\circ} 13,668^{\prime} \mathrm{W}\right)$, altitude de $840 \mathrm{~m}$, em plantas da cultivar Giombo, com 17 anos de idade e altura média de $5 \mathrm{~m}$.

A pulverização foi realizada utilizando-se um conjunto mecanizado composto de trator marca Valmet, modelo 88, e pulverizador turboatomizador marca Jacto, modelo Arbus 2000, equipado com pontas de pulverização J4, marca Jacto. Os 
Turboatomizador e repasse com pistola manual na cobertura de pulverização de agrotóxicos em caquizeiro (Diospyros kaki L.f.). 847

tratamentos utilizados foram taxa de aplicação (Alta e Baixa) e repasse com pistola manual (com e sem). A aplicação foi realizada em duas velocidades de deslocamento do equipamento, o que promoveu taxas de aplicação de 2857 e $1905 \mathrm{~L} \mathrm{ha}^{-1}$ (12 e 8L por planta, respectivamente). Para a realização do repasse, foi utilizada pistola manual, marca KO, modelo PC-06, acoplada ao mesmo equipamento. $\mathrm{O}$ posicionamento da folha em relação à planta, interna ou externa, foi considerado como um dos fatores (subparcela), assim como a superfície das folhas, abaxial e adaxial (subsubparcela). Durante a aplicação, os valores médios dos fatores climáticos foram: temperatura $22^{\circ} \mathrm{C}$, umidade relativa do ar $61 \%$ e velocidade do vento $1,5 \mathrm{~km} \mathrm{~h}^{-1}$.

Para a avaliação da cobertura, foi utilizada calda preparada com corante fluorescente (derivado de ureia/formaldeído) em água (HIMEL, 1969; TOMPKINS et al., 1983; IRLA, 1990; EVANS et al., 1994). Para dissolver o corante, foi realizada mistura prévia com fungicida Manzate (mancozeb, $800 \mathrm{~g} \mathrm{~kg}^{-1}$ de ingrediente ativo). As proporções usadas foram: 100L de água, $128,33 \mathrm{~g}$ de fungicida e $176 \mathrm{~g}$ de corante (ABI SAAB, 1996).

Após aproximadamente 15 minutos da aplicação (tempo suficiente para secagem da calda), foram coletadas, proporcionalmente à distribuição no dossel, 10 folhas na posição externa e 10 folhas na posição interna de cada planta (subparcelas). Após a coleta, cada folha foi imediatamente colocada em saco de papel devidamente identificado.

Posteriormente, em sala escura e com iluminação ultravioleta para destacar a área atingida pelo corante fluorescente, as superfícies abaxial e adaxial de cada folha (subsubparcelas) foram fotografadas com câmera digital. Cada imagem gerada foi submetida à análise pelo programa SIARCS 3.0, resultando em porcentagem de cobertura.

O delineamento experimental utilizado, em esquema fatorial, foi de blocos ao acaso, com quatro repetições e parcelas subsubdivididas, sendo os tratamentos (taxas alta e baixa de aplicação, com e sem repasse manual) nas parcelas, posição das folhas na planta na subparcela, e superfície das folhas na subsubparcelas. Os dados foram submetidos à análise de variância, complementada pelo Teste de Tukey a $5 \%$ de probabilidade de erro.

\section{RESULTADOS E DISCUSSÃO}

Houve interação significativa entre tratamentos (taxa de aplicação e repasse manual), localização na planta e superfície das folhas. Para facilitar o entendimento, a apresentação dos dados foi separada em superfície abaxial (Tabela 1) e adaxial (Tabela 2) das folhas.

A maior taxa de aplicação promoveu aumento significativo da cobertura na superfície abaxial das folhas do caquizeiro (Tabela 1), independentemente da localização destas na planta (interna ou externa).

A superfície abaxial das folhas, localizadas na parte interna da planta, teve cobertura significativamente maior quando foi feito o repasse manual. Para essa posição (superfície abaxial das folhas, localizadas na parte interna da planta), que

Tabela 1 - Média do porcentual de cobertura da superficie abaxial das folhas do caquizeiro, resultante da aplicação de agrotóxicos.

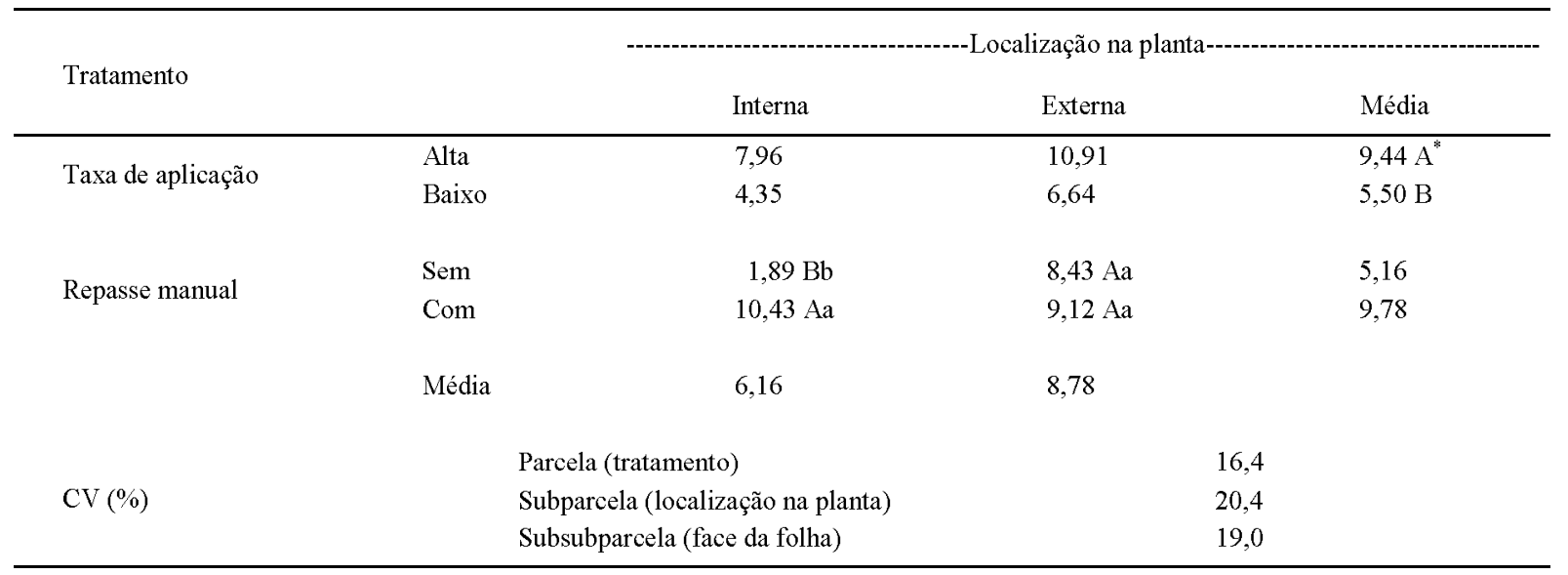

*Médias seguidas por letras iguais, maiúsculas nas colunas e minúsculas nas linhas, não diferem significativamente entre si.

Ciência Rural, v.43, n.5, mai, 2013. 
Tabela 2 - Média do porcentual de cobertura da superfície adaxial das folhas do caquizeiro, resultante da aplicação de agrotóxicos.

\begin{tabular}{|c|c|c|c|c|c|}
\hline \multirow[t]{2}{*}{ Tratamento } & & \multirow[b]{2}{*}{ Interna } & \multirow{2}{*}{\multicolumn{2}{|c|}{ Externa }} & \multirow[b]{2}{*}{ Média } \\
\hline & & & & & \\
\hline \multirow{2}{*}{ Taxa de aplicação } & Alta & $21,19 \mathrm{Aa}^{*}$ & \multicolumn{2}{|l|}{$12,10 \mathrm{Ab}$} & 16,65 \\
\hline & Baixo & $13,71 \mathrm{Ba}$ & $8,83 \mathrm{Bb}$ & & 11,27 \\
\hline \multirow{3}{*}{ Repasse manual } & Sem & $7,16 \mathrm{Ba}$ & \multicolumn{2}{|l|}{$8,06 \mathrm{Ba}$} & 7,61 \\
\hline & Com & $27,74 \mathrm{Aa}$ & \multicolumn{2}{|l|}{$12,87 \mathrm{Ab}$} & 20,31 \\
\hline & Média & 17,45 & \multicolumn{2}{|l|}{10,47} & \\
\hline \multirow{3}{*}{$\mathrm{CV}(\%)$} & \multicolumn{2}{|c|}{ Parcela (tratamento) } & \multicolumn{3}{|c|}{11,6} \\
\hline & \multicolumn{2}{|c|}{ Subparcela (localização na planta) } & \multicolumn{3}{|c|}{19,8} \\
\hline & \multicolumn{2}{|c|}{ Subsubparcela (face da folha) } & \multicolumn{3}{|c|}{8,8} \\
\hline
\end{tabular}

*Médias seguidas por letras iguais, maiúsculas nas colunas e minúsculas nas linhas, não diferem significativamente entre si.

é considerada a mais crítica para o início do ataque de pragas e doenças, o efeito do repasse manual deve ser considerado como importante alternativa no tratamento preventivo. Por outro lado, não houve efeito do repasse manual na superfície abaxial das folhas localizadas na parte externa da planta, assim como não houve diferença significativa na cobertura porcentual entre as folhas situadas nas posições externa e interna da planta.

Na superfície adaxial das folhas (Tabela 2), a maior taxa de aplicação também promoveu aumento significativo da cobertura das folhas, localizadas tanto interna quanto externamente à planta, repetindo o que ocorreu na superfície abaxial (Tabela 1). Essa observação coincide com as constatações de TOMPKINS et al. (1983) e MAITHIA (1991), em plantas de feijão vagem e café, respectivamente.

No entanto, é interessante notar que, nessa superfície (adaxial), a cobertura foi significativamente maior na posição interna da planta, independentemente da taxa de aplicação. Essa ocorrência faz supor que a velocidade do vento gerado pelo equipamento de aplicação pode ser excessiva para a condição da cultura em estudo. As gotas ultrapassariam a camada inicial de folhas (externa) e, pelo arrasto provocado pelo vento, iriam se localizar com maior intensidade nas folhas posicionadas internamente ao dossel da planta. Para esse tipo de aplicação, outro fator a considerar e que também deve ser objeto de futuros estudos, é o espectro das gotas geradas pelo equipamento utilizado. Gotas menores são mais facilmente carregadas pela corrente de vento, e também tendem a evaporar mais rapidamente, do que gotas maiores.
A heterogeneidade do alvo nos cultivos de frutíferas dificulta a aplicação uniforme nas diferentes partes das plantas, como observado por GIL et al. (1996) em videiras. Nesse caso, o uso apropriado de assistência de ar é exigido para melhorar a distribuição (MATTHEWS, 2000), considerando-se ainda que o volume de ar deve ser ajustado às circunstâncias específicas de cada aplicação (KAUL et al., 2002). Com velocidade muito alta do ar, o produto pode ser soprado através e acima do alvo (VEREECKE et al., 2000). A observação de que menor velocidade de ar, com maior taxa de aplicação, proporciona melhor cobertura também tem sido citada na literatura (RANDALL, 1971). Avaliando a deposição, HOLOWNICKI et al. (2000) encontraram maiores valores com menor fluxo de ar, num turboatomizador auto ajustável testado em frutíferas.

Quanto ao repasse manual, seu efeito foi significativo, independente da localização da folha na planta (interna ou externa). Todavia, a superfície adaxial das folhas de caquizeiro teve cobertura significativamente maior quando posicionada internamente (Tabela 2). Esse fato, aliado à menor cobertura na superfície abaxial das folhas posicionadas internamente e sem repasse manual (Tabela 1), reforça a importância do repasse, que visa a melhorar a cobertura nessa parte da planta.

\section{CONCLUSÃO}

Nas condições do experimento, o repasse manual promoveu maior cobertura porcentual na face abaxial das folhas posicionadas no interior da planta do caquizeiro, em comparação ao aumento da taxa de aplicação. 


\section{REFERÊNCIAS}

ABI SAAB, O.J.G. Avaliação de um sistema de aplicação de defensivos utilizado em videiras no Município de Londrina/ PR. 1996. 65f. Dissertação (Mestrado em Agronomia/ Energia na Agricultura) - Faculdade de Ciências Agronômicas, Universidade Estadual Paulista, SP.

BUCK, J.W. et al. Effect of postsymptom application of fungicides on urediniospore production by Puccinia triticina on wheat and $\boldsymbol{P}$. hemerocallidis on daylily. Plant Pathology, v.95, n.3, p.325330, 2011. Disponível em: <http://apsjournals.apsnet.org/doi/ abs/10.1094/PDIS-09-10-0646> Acesso em 23 de outubro de 2012. DOI: 10.1094/PDIS-09-10-0646.

CROSS, J.V. et al. Spray deposits and losses in different sized apple trees from an axial fan orchard sprayer: 3. Effects of air volumetric flow rate. Crop Protection, v.20, p.333-343, 2001. Disponível em: <http://www.sciencedirect.com/science/article/pii/ S0261219402001928> Acesso em 23 de outubro de 2012. DOI: 10.1016/S0261-2194(02)00192-8.

EVANS M.D. et al. Fluorescent spray deposit measurement via light intensified machine vision. Applied engineering in agriculture, v.10, n.3, p.441-447, 1994. Disponível em: <http://agris. fao.org/agris-search/search/display.do?f=1995\%2FUS\%2FUS95379. xml\%3BUS9512076> Acesso em 23 de outubro de 2012.

GIL E. et al. Design and verification of a moving equipment for orchard and vineyard sprayer calibration. In: INTERNATIONAL CONFERENCE ON AGRICULTURAL ENGINEERING, 1996, Madrid. Proceedings... Madrid: European Society of Agricultural Engineers, 1996. 8p. Paper N96A-141.

HIMEL, C.M. The fluorescent particle spray droplet tracer method. Journal of Economic Entomology, v.62, n.4, p.912-91 6, 1969. Disponível em: <http://www.ingentaconnect.com/content/ esa/jee/1969/00000062/00000004/art00046> Acesso em 23 de outubro de 2012.

HOLOWNICKI, R. et al. Automatic self adjusting air-jet sprayer concept for fruit trees. In: INTERNATIONAL CONFERENCE ON AGRICULTURAL ENGINEERING, 2000, Warwick. Proceedings... Warwick: European Society of Agricultural Engineers, 2000. 10p. Paper n.00-PM-053.

INSTITUTO BRASILEIRO DE GEOGRAFIA E ESTATISTICA. Lavoura permanente 2009a. Estados@. Disponível em: <http:// www.ibge.gov.br/home/estatistica/economia/pam/2009/tabelas pdf/tabela03.pdf >. Acesso em: 02 ago. 2011.

INSTITUTO BRASILEIRO DE GEOGRAFIA E ESTATISTICA. Lavoura permanente 2009b. Estados@. Disponível em: <http:// www.ibge.gov.br/estadosat/temas.php?sigla=sp\&tema=lavouraper manente2009>. Acesso em: 02 ago. 2011.

INSTITUTO BRASILEIRO DE GEOGRAFIA E ESTATISTICA Lavoura permanente 2009c. Estados@. Disponível em: <http:// www.ibge.gov.br/estadosat/temas.php?sigla=pr\&tema=lavouraper manente2009>. Acesso em: 02 ago. 2011.

IRLA, E. Essais comparatifs depulvérizateurs pour la vigne, 1989. Rapports FAT, n.382, p.1, 1990.

KAUL, P. et al. Mechanisms of distribution of plant protection products sprayed in orchards. Nachrichtenblatt des Deutschen Pflanzenschutzdienstes, v.54, n.5, p.110-117, 2002. Disponível em: <http://cat.inist.fr/?aModele=afficheN\&cpsidt=13800981> Acesso em 23 de outubro de 2012.

MAITHIA, A.S.K. Optimum volume of spray application for control of coffee leaf rust. Kenya coffee, v.56, n.662, p.1231-1238 1991. Disponível em: <http://agris.fao.org/agris-search/search/ display.do?f=1993\%2FKE\%2FKE93001.xml\%3BKE9242212> Acesso em 23 de outubro de 2012.

MATTHEWS, G.A. A review of the use of air in atomization of sprays, dispersion of droplets down wind and collection on crop foliage. Aspects of Applied Biology, v.57, p.21-27, 2000. Disponível em: <http://www.cabdirect.org/abstracts/20001106839. html> Acesso em 23 de outubro de 2012.

RANDALL, J.M. The relationships between air volume and pressure on spray distribution in fruit trees. Journal of Agricultural Engineering Research, v.16, p.1-31, 1971. Disponível em: <http://www.sciencedirect.com/science/article/pii/ S0021863471800021> Acesso em 23 de outubro de 2012. DOI: $10.1016 / \mathrm{S} 0021-8634(71) 80002-1$

TOMPKINS, F.D. et al. Effect of liquid volume, spray pressure, and nozzles arrangement on coverage of plant foliage and control of snap bean rust with chlorothalonil. Plant Disease, v.67, n.9, p.952953, 1983. Disponível em: <http://www.apsnet.org/publications/ PlantDisease/BackIssues/Documents/1983Abstracts/PD_67_952. htm> Acesso em 23 de outubro de 2012. DOI: 10.1094/PD-67-952.

VEREECKE E. et al. The air distribution generated by airassisted sprayers. In: INTERNATIONAL CONFERENCE ON AGRICULTURAL ENGINEERING, AgEng-00, 2000, Warwick. Proceedings... Warwick: European Society of Agricultural Engineers, 2000. Paper n.00-PM-071.

WILLIAMSON, S.M.; SUTTON, T.B. First report of anthracnose caused by Colletotrichum acutatum on persimmon fruit in the United States. Plant Disease, v.94, n.5, p.634-635, 2010. Disponível em: <http://www.apsnet.org/publications/plantdisease/2010/May/ Pages/94_5_634.1.aspx> Acesso em 23 de outubro de 2012. DOI: 10.1094/PDIS-94-5-0634A. 